\title{
Dengue encephalitis - clinical spectrum and outcome
}

\author{
S Manimala Rao ${ }^{*}$, MV Pradeep $^{2}$, M Dnyaneshwar $^{3}$ and Taggu Alai ${ }^{4}$ \\ *Correspondence: manimalarao@hotmail.com \\ ${ }^{1}$ Chief Intensivist, Department of Critical Care Medicine, Yashoda Hospital, Hyderabad, Andhra Pradesh, India. \\ ${ }^{2}$ Fellow, Department of Critical care medicine, Yashoda Hospital, Hyderabad, Andhra Pradesh, India. \\ 3Junior Consultant, Department of Critical Care Medicine, Yashoda Hospital, Hyderabad, Andhra Pradesh, India. \\ ${ }^{4}$ Department of Anaesthesiology, Yashoda Hospital, Hyderabad, Andhra Pradesh, India.
}

\begin{abstract}
Background: Dengue viral infection is common worldwide caused by dengue virus. Recent studies have shown dengue viral infection causing encephalitis with high morbidity and mortality. The Dengue encephalitis patients usually present with altered sensorium, elevated lab parameters and high antibody titres at the time of admission.

Aims: To compare initial lab parameters, clinical spectrum and outcome in patients of dengue fever with or without encephalitis.

Settings and design: The study was done in a multi-speciality teaching hospital. Fourty nine patients were included in the study with febrile illness and thrombocytopenia, proved to be having Dengue fever.

Material and Methods: A prospective study was conducted on 49 patients with febrile illness and thrombocytopenia proved to be having Dengue fever during a period of 6 months were included. Initial lab parameters, clinical spectrum and outcome were compared in patients with or without encephalitis.

Statistical analysis used: The database of all parameters were analysed using "STATA SOFTWARE VERSION10.0".

$\underline{\text { Results: }} 49$ cases of proven dengue fever were included in the study. Among these patients 25 patients had no encephalitis and 24 patients had encephalitis. Most common clinical features in patients without encephalitis were fever with chills and rigors and in patients with encephalitis were fever with altered sensorium (P-value $<0.05$ ). Among total 49 patients, 24 patients had encephalitis. There were no statistical difference among factors like age, sex, PCV, Platelet count, PT, and INR in these two groups (with or without encephalitis) at admission. The APTT, serum Ig-M antibodies, Ig-G antibodies, SGOT and SGPT were significantly high in encephalitis group (P-value $<0.05)$. All 25 dengue patients without encephalitis recovered completely without any complication. Among 24 dengue patients with encephalitis, 22 patients recovered completely (independent for activities of daily living), and 2 patients died (due to severe sepsis with MODS).

Conclusions: Increased incidence of dengue fever with encephalitis in the recent years, in the absence of single sensitive test for detecting dengue encephalitis, variable CSF and MRI Brain features, and associated high morbidity and mortality, this study may be helpful in focussing on early diagnosis and aggressive initial management which can influence final outcome.

Keywords: Dengue fever, encephalitis, aedes aegypti, thrombocytopenia
\end{abstract}

\section{Introduction}

Dengue viral infection is common worldwide, caused by dengue virus a member of flavivirus group in the family flaviviridae, a single stranded enveloped RNA virus. Four out of every 10 people in the world are at risk of dengue viral infection [1]. Dengue fever has varying clinical presentation ranging from asymptomatic infection to life threatening hemorrhagic fever and dengue shock syndrome [2]. Common clinical features are fever, arthralgia, headache, petechial spots, rashes and hemorrhagic manifestations. The association of dengue infection with unusual neurological manifestation was first reported by Sanguansermsri et al [3]. Complications of dengue fever are common and usually related to renal and hepatic dysfunction [4]. Numerous neurological manifestations are reported like encephalopathy, encephalitis, GuillaineBarre syndrome, transverse myelitis, acute disseminated encephalomyelitis, and myositis. These neurological complications are rare and its pathogenesis is controversial, few theories states dengue neurological manifestation is secondary to systemic manifestation (Encephalopathy), but recent evidence is in favour of dengue neurotropism, because dengue virus [5] and dengue IgM antibodies has been discovered in CSF of encephalopathy patients which suggests that dengue virus is capable of central nervous system infection.

Dengue fever associated with encephalitis has high morbidity and mortality and only few studies or case series has been published regarding dengue encephalitis [6]. This study may be useful in early detection of dengue fever patients with encephalitis using clinical features and laboratory parameters in resource limited countries which are having maximum number of dengue cases, so that early diagnosis of dengue encephalitis and timely 
Rao et al. Internal Medicine Inside 2013,

http://www.hoajonline.com/journals/pdf/2052-6954-1-8.pdf

doi: $10.7243 / 2052-6954-1-8$

Table 1. Clinical features among patients with or without encephalitis at admission.

\begin{tabular}{lccc}
\hline Variable & \multicolumn{3}{c}{ Encephalitis } \\
\hline No $(\mathbf{n}=\mathbf{2 5})$ & Yes $(\mathbf{n}=\mathbf{2 4})$ & p-value \\
\hline Sex & 43.8 Years & 39.88 years & 0.56 \\
& Males-17, & Males-14, & 0.76 \\
Fever & Females- 8 & Females-10. & \\
Headache & 25 & 24 & 0.792 \\
Chills and rigor & 12 & 20 & 0.430 \\
Altered sensorium & 16 & 18 & 0.747 \\
GCS & 2 & 24 & 0.004 \\
Seizures & $(14-15 / 15) 25$ & $(7-8 / 15) 24$ & 0.03 \\
Shock & NONE & 14 & 0.006 \\
Cough & 4 & 14 & 0.04 \\
Shortness of breath & 1 & 9 & 0.659 \\
Generalised weakness & 4 & 9 & 0.04 \\
Bleeding (Purpura/ & 12 & 14 & 0.04 \\
GI bleed/Epistaxis) & & 24 & 0.004 \\
\hline
\end{tabular}

Table 2. Initial lab parameters among patients with or without encephalitis at admission.

\begin{tabular}{lccc}
\hline Variable & \multicolumn{3}{c}{ Encephalitis } \\
\hline & No $(\mathbf{n}=\mathbf{2 5})$ & Yes $(\mathbf{n}=\mathbf{2 4})$ & p-value \\
\hline HB\% & 11.03 & 11.66 & 0.48 \\
PCV & 33.78 & 32.60 & 0.63 \\
Platelets & 53,826 & 45,400 & 0.126 \\
PT & $15.52(13.2)$ & $17.925(13.2)$ & 0.27 \\
INR & 1.55 & 1.69 & 0.68 \\
APTT & $30.95(27.8)$ & 55.02 & 0.008 \\
Serum Ig-M Antibodies & 10.4 & 45.6 & 0.004 \\
Serum Ig-G Antibodies & 16.85 & 39.89 & 0.006 \\
S. Creatinine & 0.99 & 1.82 & 0.132 \\
S.Bilirubin & 1.125 & 2.21 & 0.15 \\
SGOT & 54.8 & 205 & 0.002 \\
SGPT & 57.55 & 324 & 0.001 \\
LDH & 483 & 5289 & 0.0001 \\
S.Lactate & 16.2 & 43.7 & 0.004 \\
\hline
\end{tabular}

supportive therapy can reduce or avoid morbidity and mortality. We present a total of 49 cases of dengue fever with or without encephalitis.

\section{Material and methods}

49 patients aged between 19-65 years with febrile illness and thrombocytopenia, who were admitted in acute medical care unit, Yashoda Super-speciality Hospital
Somajiguda, Hyderabad, between May 2012 to October 2012 were included in the study.

The diagnosis of dengue fever was based on clinical features, (fever, headache or bodyache, altered sensorium in case of encephalitis or encephalopathy, hemorrhagic manifestation, jaundice, and shock) and positive serum lgM / lgG antibodies.

The encephalitis group were based on clinical features (Low GCS, altered sensorium, headache, and seizures), MRI brain findings (Hyperintense areas), EEG or CSF study (IgM and IgG antibodies, Cell count, protein and sugar) suggestive of encephalitis. These tests were not done in group of dengue fever without encephalitis (without altered sensorium) or normal GCS (due to financial constraints).

Exclusion criteria were patients with previous liver or kidney failure and recent cerebral events (stroke, meningoencephalitis), malaria, and hepatitis were excluded.

The detailed medical history, age, area of residence, and clinical features were noted. Consciousness was assessed by Glasgow coma scale (GCS), Systemic manifestations such as lymphadenopathy, hepato-splenomegaly, jaundice, Cardiac, Renal and Respiratory findings were also recorded.

The laboratory tests included complete blood examination (hemoglobin, hematocrit, blood counts, platelet counts), blood sugar, blood urea, serum creatinine, bilirubin, SGOT, SGPT, prothrombin time, INR, activated partial thromboplastin time, S.lactate, LDH, Procalcitonin, C-Reactive Protein and Dengue serum antibodies. Electrocardiogram, 2D-Echo, Chest X-ray(PA view) done in all patients, and CT scan and/ or MRI brain, Cerebrospinal fluid analysis (analyzed for protein, sugar, cells, fungal and bacterial smear and culture) and Electroencephalogram were carried out in patients with altered sensorium.

Patients were conservatively managed as per WHO guidelines. Outcome was defined at 1 month into complete (independent for activities of daily living), partial (dependent for activities of daily living) and poor (bed ridden) [7].

\section{Results}

49 cases of proven dengue fever were included in the study. Among these patients 25 patients had no encephalitis and 24 patients had encephalitis.

The clinical features between patients of two groups at admission are shown in Table 1. Most common clinical features in patients without encephalitis were fever with chills and rigors and in patients with encephalitis were fever with altered sensorium. Clinical features like altered sensorium, seizures, shock, generalised weakness and shortness of breath were significant in encephalitis group ( $P$-value $<0.05)$.

The initial lab parameters between patients of two groups at admission are shown in Table 2. Among total 49 patients, 24 patients had encephalitis. There were no statistical difference among factors like $\mathrm{Hb} \%$, PCV, Platelet count, PT, and INR in these two groups (with or without encephalitis) at admission. The APTT, serum Ig-M antibodies, Ig- $G$ antibodies, SGOT and SGPT were significantly high in encephalitis group 
Rao et al. Internal Medicine Inside 2013,

(P-value < 0.05).

Among encephalitis patient group all 24 patients had altered sensorium (GCS: 7-8/15) requiring intubation and mechanical ventilator support during their stay in the hospital. All 24 encephalitis patients had severely deranged liver enzymes (SGOT, SGPT), coagulopathy (Raised PT, INR, APTT), severe thrombocytopenia and high antibody titres (lg-M and Ig-G), 7 patients required CRRT for Acute Kidney Injury for short period of time, 14 patients had generalized seizures, 3 patients had ARDS. 16 patients showed features of encephalopathy on EEG. CSF study showed high protein and normal cell counts among 15 patients and normal protein among remaining patients, In our study CSF IgM / lgG done only in 7 patients with encephalitis and lgM was positive in only 1 patient (CSF study was not done in non encephalitis patients and remaining encephalitis patients). MRI Brain study showed Bilateral hyperintensities in temporal lobes in 9 patients, thalamus in 3 patients, pons in 1 patient, and remaining patients had normal MRI findings.

All 25 dengue patients without encephalitis recovered completely without any complications during the course of stay. Among 24 dengue patients with encephalitis, 22 patients recovered completely at the end of 1 month (independent for activities of daily living), and 2 patients died (due to severe sepsis with MODS).

\section{Discussion}

Dengue viral infection is common worldwide, caused by dengue virus a member of flavivirus group in the family flaviviridae, a single stranded enveloped RNA virus. Dengue is endemic to over 100 countries and approximately 2.5 billion people are at risk. It is estimated that 50-100 million infections and 25,000 fatalities occur worldwide every year. World health organization (WHO) surveillance shows that global incidence is increasing [8]. The primary vector is the mosquito Aedes Aegypti, found in abundance from latitudes 35 degree North to 35 degree South.

Dengue fever has varying clinical presentation ranging from asymptomatic infection to life threatening hemorrhagic fever and dengue shock syndrome. Common clinical features are fever, arthralgia, headache, petechial spots, rashes and hemorrhagic manifestations. The association of dengue infection with unusual neurological manifestation was first reported by Sanguansermsri et al.

Complications of dengue fever are common and usually related to renal and hepatic dysfunction. In our study all 25 patients without encephalitis had mild hepatic or renal dysfunction, where as all 24 encephalitis patients had severely deranged liver enzymes (SGOT, SGPT), coagulopathy (Raised PT, INR, APTT), severe thrombocytopenia and high antibody titres (Ig-M and Ig-G).

Patients were managed conservatively as per WHO guidelines. In the patients with hemorrhagic diathesis, platelet concentrate and/or fresh frozen plasma were administered as indicated. All patients with low GCS $(n=24)$ were intubated to protect the airway and majority of them extubated after 5-7 days. Tracheostomy was done in four patients among them 2 patients were de-cannulated and other 2 patients were on prolonged ventilation who died later due to refractory septic shock and MODS due to secondary bacterial sepsis (Procalcitonin and CRP were high in these two patients). CRRT done for patients with AKI (Acute kidney injury) having oliguria/anuria, Pulmonary edema, Hyperkalemia and severe metabolic acidosis.

Numerous neurological manifestations are reported like encephalopathy, encephalitis, Guillaine-Barre syndrome [9], transverse myelitis, acute disseminated encephalomyelitis [10], and myositis [11]. These neurological complications are rare and its pathogenesis is controversial, few theories states dengue neurological manifestation is secondary to systemic manifestation (Encephalopathy), but recent evidence is in favour of dengue neurotropism, because dengue virus [12] and dengue IgM antibodies [13] has been discovered in CSF of encephalopathy patients which suggests that dengue virus is capable of central nervous system infection.

In our study the features of encephalitis (headache, altered sensorium, and seizures) in majority of patients were seen more commonly in secondary dengue infection and after 5-7 days of onset of fever. Among the four dengue serotypes ( DEN-1 to DEN-4) DEN-2 and DEN-3 have highest propensity to neurological complications $[14,15]$.

Our study had more number of Dengue encephalitis patients, probably due to tertiary hospital (Referral hospital) or may be due to antigenic variation of virus. Cam BV et al., and Hendarto SK et al., have reported the encephalopathy incidence ranging from $0.5 \%$ to $6.2 \%$. Kankirawatana et al., found that $18 \%$ of children with suspected Encephalitis in Thai hospital were found to have dengue infection. Misra et al., described 11 encephalopathy patients with confirmed dengue infection, and they demonstrated lymphocytic pleocytosis in CSF.

The patients who died in encephalitis group ( 2 out of 24 patients), both of them presented with - low GCS, seizures, headache and upper GI bleed. Hence patients with dengue fever with predominance of these clinical features and severely deranged lab parameters are probably manifesting encephalitis which has high morbidity and mortality unless diagnosed early and treated accordingly.

The outcome in dengue encephalitis depends upon how early the diagnosis is made and managed aggressively in due course of time because we can anticipate the course of encephalitis and its complications. Misra et al., proposed that Encephalitis lies at the severe end of the spectrum of dengue infection, but others reported a more benign disease [16].

In case of dengue Encephalitis diagnosis can be made either by detection of virus in CSF (viral culture / PCR) or immune response by the body (Ig-M antibodies in CSF). The gold standard method is viral culture which is difficult and time consuming. Regarding CSF IgM and IgG antibodies, 
Rao et al. Internal Medicine Inside 2013,

Puccioni-Sohler $\mathrm{M}$ et al., [17] and Cristiane Nascimento Soares [18] have shown that these antibodies can be seen in CSF but the absence will not rule out encephalitis. In our study CSF IgM and IgG done only in 7 patients with encephalitis and IgM was positive in only 1 patient (CSF study was not done in remaining patients as the sensitivity of this test found to be very low and was thought to be financial burden for those patients). CSF study in encephalitis group showed high protein and normal cell counts among 15 patients and normal protein among remaining patients but serum $\lg \mathrm{M}$ and $\lg \mathrm{G}$ were positive in all patients.

Singh et al., [19], found specificity of PCR assay to be $100 \%$ and sensitivity of $70 \%$ when sample are taken in first 5 days of fever.

Brain imaging- MRI is the modality of choice which shows the findings consistent with viral encephalitis include cerebral edema, white matter changes, necrosis and brain atrophy. Encephalitis features in brain (Hyperintense areas) can be seen in global pallidus, temporal lobes [20,21], thalamus [22], hippocampus [23], pons, and spinal cord [24]. In our study, MRI Brain study showed Bilateral hyperintense areas in temporal lobes in 9 patients, thalamus in 3 patients, pons in 1 patient, and remaining patients had normal MRI findings (MRI Brain was not done in non encephalitis patients).

All 25 dengue patients without encephalitis recovered completely without any complications during the course of stay. Among 24 dengue patients with encephalitis, 22 patients recovered completely at the end of 1 month (independent for activities of daily living), and 2 patients died (due to severe sepsis with MODS).

The limitation of our study is that we could not do CSF study, and MRI imaging in proven dengue patients without encephalitis (as thought not essential). We could do only serum Ig-M and Ig-G antibodies. CSF culture or PCR for viral detection in CSF was not done. But all patients proved to be having dengue serum antibodies are managed conservatively according to WHO guidelines.

More research is necessary for the changing trend of host immunological response and dengue viral characteristics as more patients with dengue viral infection in recent years are presenting with encephalitis.

\section{Conclusion}

Increased incidence of dengue fever with encephalitis in the recent years, in the absence of single sensitive test for detecting dengue encephalitis, variable CSF and MRI Brain features, and associated high morbidity and mortality, this study may be helpful in focussing on early diagnosis and aggressive initial management which can influence final outcome.

\section{Competing interests}

The authors declare that they have no competing interests.

Authors' contributions
\begin{tabular}{|l|c|c|c|c|}
\hline Authors' contributions & RMS & MVP & MD & TA \\
\hline Research concept and design & $\sqrt{ }$ & -- & $\sqrt{ }$ & -- \\
\hline Collection and/or assembly of data & $\sqrt{ }$ & $\sqrt{ }$ & -- & -- \\
\hline Data analysis and interpretation & $\sqrt{ }$ & -- & $\sqrt{ }$ & -- \\
\hline Writing the article & $\sqrt{ }$ & $\sqrt{ }$ & -- & -- \\
\hline Critical revision of the article & $\sqrt{ }$ & $\sqrt{ }$ & -- & -- \\
\hline Final approval of article & $\sqrt{ }$ & -- & $\sqrt{ }$ & -- \\
\hline Statistical analysis & $\sqrt{ }$ & $\sqrt{ }$ & -- & $\sqrt{ }$ \\
\hline
\end{tabular}

\section{Acknowledgement}

We gratefully acknowledge the internal medicine physicians respiratory technicians, and nurses and hospital administrators for their valuable support.

\section{Publication history}

EIC: Fabio Angeli, University of Perugia, Italy.

Received: 28-May-2013 Revised: 24-Jul-2013

Re-Revised: 14-Sep-2013 Accepted: 20-Sep-2013

Published: 01-Oct-2013

\section{References}

1. World health organization. Dengue hemorrhagic fever; diagnosis, treatment, prevention and control. Geneva: WHO; 1997. I Website

2. Koley TK, Jain S, Sharma H, Kumar S, Mishra S, Gupta MD and Goyal AK. Dengue encephalitis. J Assoc Physicians India. 2003; 51:422-3. I PubMed

3. Sanguansermsri T, Poneprasert B, Phornphutkul B, et al. Acute encephalopathy associated with dengue infection. Bangkok: SEAMEO TROPMED1976: 10-11.

4. Monath TP. Dengue: the risk to developed and developing countries. Proc Natl Acad Sci U S A. 1994; 91:2395-400. | Article | PubMed Abstract | PubMed FullText

5. Solomon T, Dung NM, Vaughn DW, Kneen R, Thao LT, Raengsakulrach B, Loan HT, Day NP, Farrar J, Myint KS, Warrell MJ, James WS, Nisalak A and White NJ. Neurological manifestations of dengue infection. Lancet. 2000; 355:1053-9. | Article | PubMed

6. Varatharaj $A$. Encephalitis in the clinical spectrum of dengue infection. Neurol India. 2010; 58:585-91. | Article | PubMed

7. Misra UK, Kalita J, Jain SK and Mathur A. Radiological and neurophysiological changes in Japanese encephalitis. J Neurol Neurosurg Psychiatry. 1994; 57:1484-7. | Article | PubMed Abstract | PubMed FullText

8. NaTHNaC. Travel Health Information Sheets: Dengue fever. | Pdf

9. Sulekha C, Kumar S and Philip J. Guillain-Barre syndrome following dengue fever. Indian Pediatr. 2004; 41:948-50. | PubMed

10. Yamamoto Y, Takasaki T, Yamada K, Kimura M, Washizaki K, Yoshikawa K, Hitani A, Nakamura T and Iwamoto A. Acute disseminated encephalomyelitis following dengue fever. J Infect Chemother. 2002; 8:175-7. | Article | PubMed

11. Misra UK, Kalita J, Syam UK and Dhole TN. Neurological manifestations of dengue virus infection. J Neurol Sci. 2006; 244:117-22. | Article | PubMed

12. Cam BV, Fonsmark L, Hue NB, Phuong NT, Poulsen A and Heegaard ED. Prospective case-control study of encephalopathy in children with dengue hemorrhagic fever. Am J Trop Med Hyg. 2001; 65:848-51. | Article I PubMed

13. Kankirawatana P, Chokephaibulkit K, Puthavathana P, Yoksan S, Apintanapong $S$ and Pongthapisit V. Dengue infection presenting with central nervous system manifestation. J Child Neurol. 2000; 15:544-7. Article I PubMed

14. Gulati S and Maheshwari A. Atypical manifestations of dengue. Trop Med Int Health. 2007; 12:1087-95. | Article | PubMed 
Rao et al. Internal Medicine Inside 2013,

http://www.hoajonline.com/journals/pdf/2052-6954-1-8.pdf

15. Lum LC, Lam SK, Choy YS, George R and Harun F. Dengue encephalitis: a true entity? Am J Trop Med Hyg. 1996; 54:256-9. I Article I PubMed

16. Kularatne SA, Pathirage MM and Gunasena S. A case series of dengue fever with altered consciousness and electroencephalogram changes in Sri Lanka. Trans R Soc Trop Med Hyg. 2008; 102:1053-4. | Article | PubMed

17. Puccioni-Sohler M, Soares CN, Papaiz-Alvarenga R, Castro MJ, Faria LC and Peralta JM. Neurologic dengue manifestations associated with intrathecal specific immune response. Neurology. 2009; 73:1413-7. | Article | PubMed

18. Cristiane Nascimento Soares. Neurological manifestations of dengue infection: clinical. characteristics and cerebrospinal fluid analysis. Neurologia 2005;63. | Article

19. Singh K, Lale A, Eong Ooi E, Chiu LL, Chow VT, Tambyah P and Koay ES. A prospective clinical study on the use of reverse transcriptionpolymerase chain reaction for the early diagnosis of Dengue fever. $J$ Mol Diagn. 2006; 8:613-6; quiz 617-20. | Article | PubMed Abstract | PubMed FullText

20. Muzaffar J, Venkata Krishnan P, Gupta N and Kar P. Dengue encephalitis: why we need to identify this entity in a dengue-prone region. Singapore Med J. 2006; 47:975-7. | Pdf | PubMed

21. Wasay M, Channa R, Jumani M, Shabbir G, Azeemuddin M and Zafar A. Encephalitis and myelitis associated with dengue viral infection clinical and neuroimaging features. Clin Neurol Neurosurg. 2008; 110:635-40. | Article | PubMed

22. Kamble R, Peruvamba JN, Kovoor J, Ravishankar S and Kolar BS. Bilateral thalamic involvement in dengue infection. Neurol India. 2007; 55:418-9. | Article | PubMed

23. Yeo PS, Pinheiro L, Tong P, Lim PL and Sitoh YY. Hippocampal involvement in dengue fever. Singapore Med J. 2005; 46:647-50. | Pdf | PubMed

24. Soares CN, Faria LC, Peralta JM, de Freitas MR and Puccioni-Sohler $M$. Dengue infection: neurological manifestations and cerebrospinal fluid (CSF) analysis. J Neurol Sci. 2006; 249:19-24. | Article | PubMed

\section{Citation:}

Rao SM, Pradeep M, Dnyaneshwar M and Alai T. Dengue encephalitis - clinical spectrum and outcome. Intern Med Inside. 2013; 1:8.

http://dx.doi.org/10.7243/2052-6954-1-8 